\title{
Experimental induction of neurofibromatosis in bicolor damselfish
}

\author{
Michael C. Schmale* \\ Division of Marine Biology and Fisheries, Rosenstiel School of Marine and Atmospheric Sciences, University of Miami, \\ 4600 Rickenbacker Causeway, Miami, Florida 33149, USA
}

\begin{abstract}
Damselfish neurofibromatosis (DNF) is a neoplastic disease affecting the peripheral nervous system and chromatophores of bicolor damselfish Pomacentrus partitus on Florida (USA) reefs. This report presents the results of transmission experiments using intramuscular and intraperitoneal injection of a variety of tumor preparations to induce DNF. Neurofibromas developed within 1 yr of injection in $76 \%$ of fish injected with homogenates of spontaneously occurring tumors with earliest tumor development occurring at 5 to $6 \mathrm{wk}$ post-injection. Cell-free transmission using $0.2 \mu \mathrm{m}$ filtrates of DNF tumor homogenates was observed, demonstrating that the agent responsible for DNF is subcellular. Four out of five tumor cell lines established from neurofibromas were found to be tumorigenic when injected, while cells cultured from normal fish had no effect, indicating that the etiologic agent of DNF was present and infectious in tumor cell lines but not in cell lines or primary cultures derived from healthy fish. Serial passages of tumors were conducted through 19 passage generations in vivo. A comparison of the cumulative time to tumor distributions showed that most experiments beyond the third serial passage yielded significantly faster tumor development than was obtained by passaging from spontaneous tumors. Induced tumors exhibited more localized growth patterns and were typically more rapidly growing and more rapidly lethal than spontaneous tumors. In contrast to the differences in growth patterns observed, the histological phenotype of the spontaneous, wild-type DNF lesions was indistinguishable from that of the induced tumors obtained from serial passages as well as in experiments involving cell-free filtrates and cultured tumor cells. These observations indicate that the process of neoplastic transformation is relatively stable in this system, with little or no phenotypic drift caused by these manipulations. Naturally occurring, transmissible cancers that arise from cells of the central or peripheral nervous system or from chromatophores have not been previously identified. Thus, DNF is a unique disease and may provide an important model for investigating this rather unique form of viral oncogenesis.
\end{abstract}

KEY WORDS: Tumor Neurofibromatosis - Damselfish Transmission

\section{INTRODUCTION}

Damselfish neurofibromatosis (DFN) is a neoplastic disease affecting the peripheral nervous system and chromatophores of bicolor damselfish Pomacentrus partitus on Florida (USA) reefs (Schmale 1991). The disease consists of multiple neurofibromas and neurofibrosarcomas, many of which are pigmented (Schmale et al. 1983, 1986). The prevalence of DNF in natural populations is positively correlated with population density and cases are significantly clustered in space,

\footnotetext{
•E-mail:mschmale@rsmas.miami.edu
}

suggesting a horizontally transmitted etiologic agent (Schmale 1991). It has previously been demonstrated that DNF can be transmitted in the laboratory by subcutaneous and intraperitoneal injection of tumor homogenates into healthy fish (Schmale \& Hensley 1988).

A wide variety of transmissible neoplasms have been identified in mammals and birds with etiologic agents generally identified as DNA or RNA viruses (Tooze 1980, Weiss et al. 1982). Several neoplasms in fish have been suspected of being associated with viruses and/or of being transmitted horizontally. However, conclusive transmission experiments have only been conducted with a few of these tumors from which viruses have been isolated. Retroviruses have been shown to be the 
etiologic agents for lymphosarcomas in pike and other esocids, dermal sarcoma in walleye and plasmacytoid leukemia in coho salmon (Papas et al. 1976, Martineau et al. 1991, Eaton \& Kent 1992). Epidermal papillomas in masu salmon and in carp have been shown to be caused by herpesviruses (Anders \& Yoshimizu 1994).

The majority of infectious neoplasms in animals are leukemias, lymphomas and sarcomas. As yet naturally occurring, transmissible cancers that arise from cells of the central or peripheral nervous system or from chromatophores have not been identified. Thus, DNF is a unique disease and may provide an important opportunity to identify an etiologic agent that affects 2 neuroectodermal cell types, Schwann cells and chromatophores. Here we describe the transmission of DNF using cell-free filtrates of tumor homogenates and cells from DNF-derived cell lines. In addition, we report on serial transmission experiments demonstrating that repeated in vivo passaging of tumors results in significant acceleration of tumorigenesis.

\section{MATERIALS AND METHODS}

Fish specimens. Bicolor damselfish Pomacentrus partitus were collected on reefs throughout South Florida which had previously been surveyed to determine disease prevalence rates (Schmale 1991). Fish were maintained in either recirculating or flow-through marine aquaria of $\geq 351$ at 20 to $30^{\circ} \mathrm{C}$. Normal fish were observed in the laboratory for several weeks following capture to confirm initial observations that they lacked any signs of DNF. Previous studies demonstrated that tumors appeared significantly more rapidly in juvenile fish (total length less than $60 \mathrm{~mm}$; Schmale \& Hensley 1988). All data presented here are from injection of juvenile fish, ranging in size from 30 to $60 \mathrm{~mm}$ total length.

Injection protocol. Tumorigenicity of various preparations of tumor and normal tissues and cells was assessed by injecting these into normal, healthy, juvenile bicolor damselfish. Fish were lightly anesthetized with MS-222 (tricaine methanesulfonate, Argent Labs), $120 \mathrm{mg} \mathrm{ml}^{-1}$, before being injected intramuscularly at 2 sites on opposite sides of the body. Both sites were located several $\mathrm{mm}$ ventral to the lateral line at a depth of 1 to $2 \mathrm{~mm}$ below the dermis. The 2 injection sites were termed anterior right (AR) and posterior left (PL), with AR located just behind the head region, in an area above the pectoral fin, while PL was located more posteriorly. In several of the earlier experiments, fish were also injected intraperitoneally. However, this technique produced significantly lower tumor yields and, unless otherwise specified, analyses were based on results from $A R$ and PL sites only. Approximately 20 to
$50 \mu$ of one of the tumor materials described below was injected at each site, with volume based on the size of the fish.

Preparation of tissue extracts and cells. Tumors were initially obtained from fish with DNF collected in the wild. Fish were euthanized using MS-222 at a dosage of $180 \mathrm{mg} \mathrm{l}^{-1}$ and tumors collected using aseptic procedures. Material from each experiment was generally derived from 1 tumor and, with the exception of the first experiment involving 2 tumored fish, tumors were not pooled. Tumors were homogenized in Hank's Balanced Salt Solution without $\mathrm{Ca}^{+2}$ or $\mathrm{Mg}^{+2}$ (HBSS, Gibco) or Leibovitz L-15 medium (L-15, Gibco) at a concentration of $80 \mathrm{mg} \mathrm{ml}^{-1}$ using a Ten Broek or Duall glass homogenizer on ice as described previously (Schmale \& Hensley 1988). The concentration of intact. cells in each homogenate was counted using a hemocytometer. Tumors that resulted from injection of homogenate obtained by this protocol were harvested and prepared in an identical fashion for injection into healthy fish to propagate the tumor in vivo. These experiments were termed serial passages and numbered sequentially (i.e. passage $1,2,3, \ldots$ ) to denote the number of times the tumor was passaged in vivo following the original passage from a spontaneously tumored fish (denoted as passage 0). Controls were performed by injection of muscle tissue homogenates prepared from normal fish at $80 \mathrm{mg} \mathrm{ml}^{-1}$.

Cell-free filtrates were produced by centrifugation of tumor homogenate at $300 \times g$ for $20 \mathrm{~min}$ at $4^{\circ} \mathrm{C}$ followed by pre-filtration through an $0.8 \mu \mathrm{m}$ syringe filter and final filtration through a $0.2 \mu \mathrm{m}$ cellulose acetate filter (either Millipore, Schleicher \& Schuell, Centricon or Costar). Aliquots of all filtrates were stained with trypan blue and examined at $400 \times$ to assure that they were cell-free.

Cultured cells used for injections were obtained from 5 tumor cell lines and an embryo cell line (derived from embryos obtained from normal fish) established previously and primary cultures obtained from enzymatic dissociation of fins from normal fish. The tumor cell lines were composed entirely of neoplastic Schwann cells as described previously (Schmale et al. 1994), while the other cultures consisted of a mixture of cell types including fibroblasts, epithelial cells, chromatophores and Schwann cells. All cultures were main. tained in L-15 with $10 \%$ fetal bovine serum and antibiotics as described previously (Schmale et al. 1994). Cells were collected from cultures immediately prior to being injected. Cells were harvested by treatment with $0.25 \%$ trypsin (Gibco) in HBSS for 10 to 20 min, followed by rinsing and/or scraping cells from the flask, inhibition of trypsin activity by $10 \%$ horse or fetal bovine serum, pelleting of cells at $800 \times g$ for $10 \mathrm{~min}$, resuspension in several $\mathrm{ml}$ of HBSS and counting in a 
hemocytometer. Cell concentrations were adjusted and injections were conducted using concentrations of 1 to $10 \times 10^{6}$ cells $\mathrm{ml}^{-1}$

Data collection. Injected fish were observed for the presence of externally visible lesions at weekly intervals for $8 \mathrm{wk}$ following injection and at monthly intervals thereafter for at least 1 yr. The use of a 1 yr time interval was based on experience from previous studies run for longer periods that had indicated that the majority of juvenile fish that develop tumors in laboratory experiments do so within $1 \mathrm{yr}$ of injection (Schmale \& Hensley 1988). Fish were euthanized at 12 mo post-injection or at any time following tumor development for harvest of tissues or if they exhibited impaired feeding or swimming behavior.

The only variable measured in the present study to estimate tumorigenesis was time to appearance of tumors. Time of tumor development was scored at the appearance of a small hyperpigmented spot or raised area on the skin. However, scoring such an event as a positive tumor was dependent on the subsequent development of a conspicuous tumor at that site. Any tumor appearing on the body more than $1 \mathrm{~cm}$ from an injection site or anywhere on the fins was scored as being an 'other' site. Attempts to measure tumor sizes in live fish were abandoned due to the difficulty of estimating the extent of subcutaneous growth of the tumors beyond the externally visible lesions. Thus, we were unable to estimate tumor growth rates. However, tumors from a subset of fish were dissected and weighed to determine a range of tumor weights relative to body weights. A subset of fish was collected for histological analyses at various stages of tumor development. However, no attempt was made to systematically survey the histology of tumors in each experiment or to screen asymptomatic fish for small tumors.

Data analysis. Several parameters were used in combination to compare rates of tumorigenesis between experiments or groups of experiments. This combination was used because no single parameter could completely reflect the pattern of tumor development. The maximal rate of tumorigenesis in an experiment was estimated by the time to first tumor, which was defined as the minimum time required for a tumor to appear in any fish in a given group. The maximum and mean times of tumorigenesis could not be directly measured due to the necessity of terminating an experiment after a fixed interval and thereby truncating the right side of the time-to-tumor distribution (unless all fish in a group had developed tumors within the 1 yr interval). In lieu of a mean time to tumor, the time at which $50 \%$ of the fish in an experimental group had developed tumors was used. Another measure of overall tumorigenesis used in a group was the percentage of fish that had developed tumors by the end of the 1 yr observa- tion period. Finally, a cumulative frequency distribution was plotted for each group and these curves were compared using the log-rank statistic with significant difference recognized at $p<0.05$ (Elandt-Johnson \& Johnson 1980). Fish that died or were sacrificed before appearance of tumors at less than 1 yr post-injection were considered to be censored observations. The cumulative percentage of fish developing tumors at any given time interval was calculated after correcting for changes in the denominator due to censored observations during that interval.

\section{RESULTS}

The results of injection experiments utilizing homogenates of spontaneous tumors, cultured cells and $0.2 \mu \mathrm{m}$ filtrates of homogenates of spontaneous as well as induced tumors are shown in Table 1. Tumors developed within 1 yr of injection in $76 \%$ of fish injected with homogenates of spontaneously occurring tumors, with earliest tumor development occurring at 5 to 6 wk post-injection (Table 1, Fig. 1). Several of the most rapidly appearing tumors in these initial experiments were chosen for serial passage as described below. These initial experiments utilizing donors with spontaneous tumors were termed passage 0 or Group 0 to distinguish them from subsequent passages derived from experimentally induced tumors. None of the 7 fish injected with homogenates prepared from muscle of 2 normal fish developed tumors

Cell-free transmission of DNF tumors was observed in 7 of 8 experiments conducted using $0.2 \mu \mathrm{m}$ filtrates of tumor homogenates. The proportion of fish developing tumors within $1 \mathrm{yr}$ of injection with cell-free filtrates was $9.1 \%$ for experiments using spontaneous tumors and $65.2 \%$ when experimentally induced tumors were used as donors (Table 1, Fig. 1). These data demonstrate that the agent responsible for DNF is subcellular. Rates of tumor development using filtrates of induced tumors were slightly, but not significantly slower than those from experiments based on crude homogenates of spontaneous tumors (log-rank test; $p>0.05$ ). Tumor development in experiments involving filtrates of spontaneous tumors was much slower than in all other protocols; however, log-rank analysis was precluded by the very low number of fish developing tumors in the year following injection. Yields varied greatly between experiments, with optimal tumor development occurring when using induced tumors as donors and Costar tangential flow filters (data not shown). These filters were somewhat less prone to clogging and thus may have been less likely to retain particles smaller than the specified pore size due to occlusion of the pores. The role of tumor type in deter- 
Table 1. Pomacentrus partitus. Incidence of DNF in fish injected intramuscularly with unfiltered or cell-free tissue homogenates or cultured cells. All statistics based on cumulative proportion of fish developing tumors in each group

\begin{tabular}{|c|c|c|c|c|c|}
\hline \multirow[t]{2}{*}{ Experiment type } & \multirow[t]{2}{*}{$\begin{array}{l}\text { Sample size } \\
\text { fish (trials) }\end{array}$} & \multicolumn{2}{|c|}{ Time to tumor (wk) } & \multicolumn{2}{|c|}{$\begin{array}{l}\text { Yield of tumored fish, } \\
1 \text { yr post-injection }\end{array}$} \\
\hline & & First tumor & $50 \%$ tumored & $\%$ & $N\left(\right.$ censored $\left.^{\mathrm{b}}\right)$ \\
\hline \multicolumn{6}{|l|}{ Unfiltered tissue homogenates } \\
\hline Spontaneous tumor & $43(7)$ & 5.1 & 32.7 & 76.1 & $32(1)$ \\
\hline Normal tissue & $7(2)$ & - & - & 0 & $0(0)$ \\
\hline \multicolumn{6}{|l|}{$0.2 \mu \mathrm{m}$ filtered homogenates } \\
\hline Spontaneous tumors & $12(2)$ & 21.1 & _- & 9.1 & $1^{c}(1)$ \\
\hline Induced tumors & $33(6)$ & 9.6 & 35.1 & 65.2 & $21^{d}(1)$ \\
\hline \multicolumn{6}{|l|}{ Cells from cultures } \\
\hline Tumor cell lines & $40(7)$ & 3.1 & 20.5 & 79.1 & $26(9)$ \\
\hline Normal cell lines & $15(2)$ & - & - & 0 & $0(1)$ \\
\hline Primary normal cell cultures & $18(2)$ & - & - & 0 & $0(2)$ \\
\hline \multicolumn{6}{|c|}{$\begin{array}{l}\text { "Total number of fish injected in all trials and the number of replicate trials conducted. Cultures for cell culture trials were } \\
\text { derived from either of } 2 \text { tumor cell lines ( } 88-503 \text { and 89-05), } 1 \text { normal cell line (FE1) or } 2 \text { normal primary cultures, depending } \\
\text { on the group } \\
{ }^{b} \text { Fish that died or were sacrificed during the year without developing tumors } \\
\text { c An additional fish in this group was observed to develop tumors beyond the } 1 \text { yr observation period (at } 60 \text { wk post-injection) } \\
\text { dFour additional fish in this group were observed to develop tumors beyond the } 1 \text { yr observation period (at } 58 \text { to } 64 \text { wk } \\
\text { post-injection) }\end{array}$} \\
\hline
\end{tabular}

mining these yields could not be evaluated as homogenates of spontaneous tumors were not evaluated using the Costar filters.

DNF was also induced in injection experiments utilizing 4 of the 5 tumor cell lines established from DNF tumors (88-503, 89-05, 86-583 and 89-734). The remaining tumor line $(92-16)$ did not produce any

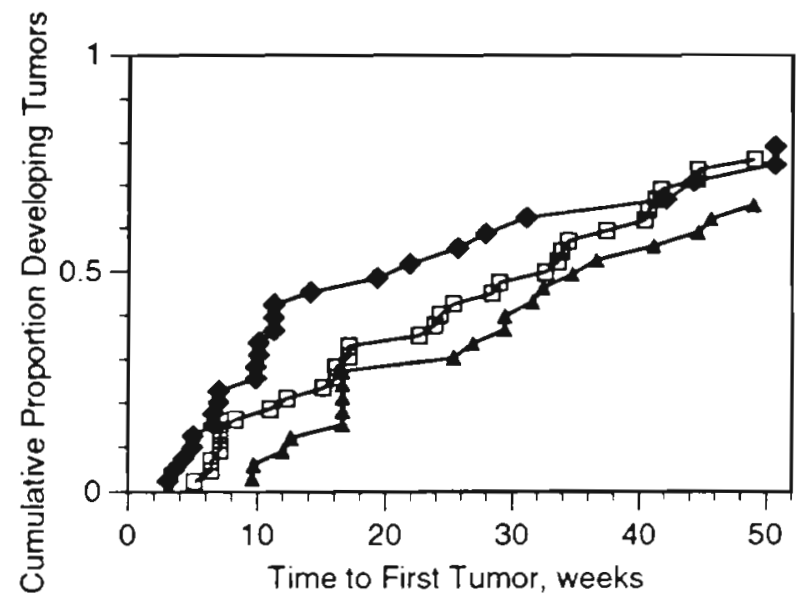

Fig. 1 Pomacentrus partitus. Cumulative proportion of fish developing tumors following injection with unfiltered homogenate of spontaneous tumors ( $\square$ ), cell-free filtrate of experimentally induced tumors $(\mathbf{\Delta})$ or cells from tumor cell-line cultures $(\bullet)$. There were no significant differences observed in rates of tumor development between these groups $(\mathrm{p}>0.05$, log-rank statistic; experiments involving cell-free filtrates of spontaneous tumors not included in analysis due small to sample sizel tumors within 8 mo of injection in 6 fish. Of these lines, 3 are from experimentally induced tumors (88-503, 86-583 and 89-734) while 2 are from spontaneous tumors (89-05 and 92-16; see Schmale et al. 1994). For analysis of tumor development rates, 6 experiments using the 88-503 cell line were combined with 1 using the 89-05 line (Table 1, Fig. 1). Experiments involving the other 3 tumor cell lines had not been observed long enough to permit inclusion in this analysis. Although tumors induced by injection of cultured tumor cells appeared somewhat faster than those in experiments using homogenates of spontaneous tumors or cell-free filtrates of induced tumors, these differences were not significant (Fig. 1; log-rank test, $p>0.05$ ). Neither primary cell cultures derived from the skin of normal fish nor cell lines derived from embryos with normal parents were tumorigenic (Table 1). These experiments demonstrated that the etiologic agent of DNF was present and infectious in tumor cell lines but not in cell lines or primary cultures derived from healthy fish.

Induced DNF tumors were serially passaged in vivo in an attempt to select for more rapidly growing tumors and to determine if the phenotype of these tumors remained stable after many passages. Nineteen serial passages involving a total of 293 fish were observed for at least 12 mo and included in analysis of tumor development rates (Fig 2A). Five additional passages, up to passage 23, were conducted and yielded tumors. However, insufficient observation time had elapsed to allow inclusion of these later data in these analyses. The 19 serial passages were combined into 3 groups based on 


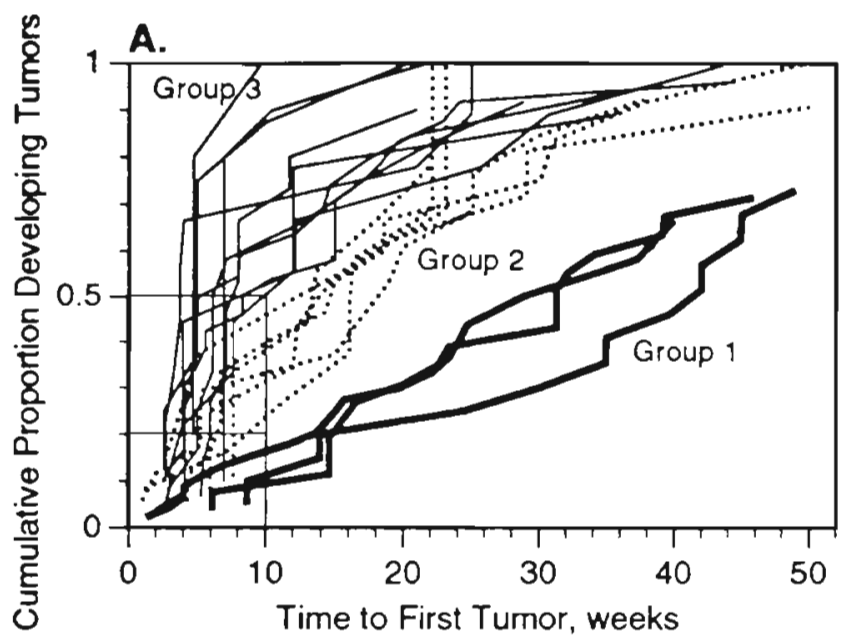

B.

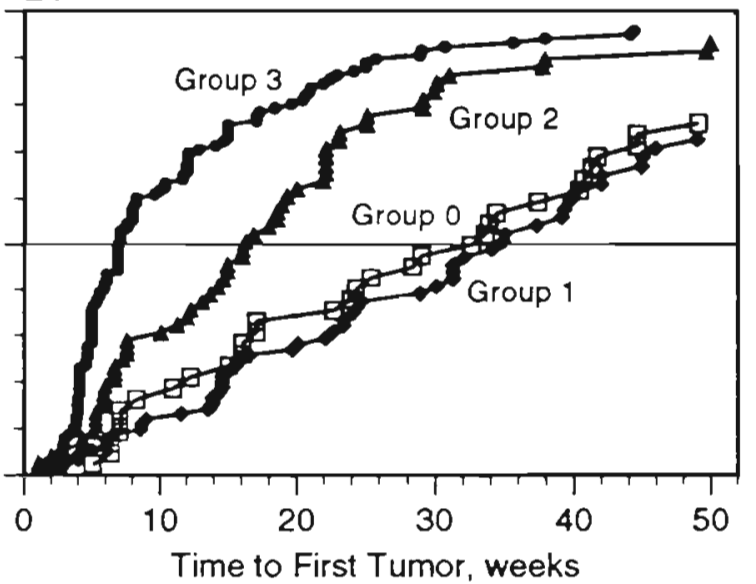

Fig. 2. Pomacentrus partitus. Serial passages of experimentally induced tumors in vivo. Cumulative proportion of fish developing tumors in 19 sequential passages using unfiltered tumor homogenates. (A) Survival curves for individual passages showing proposed groupings based on \% individuals with tumors at $10 \mathrm{wk}$ post-injection (Group 1: thick lines; Group 2: dashed lines; Group 3: thin lines). (B) Survival curves combined into 3 groups ( : Group 1; $\Delta$ : Group 2; : Group 3; see Table 2 for lists of passages included in each group). Comparison of these groups and the initial tumors derived from spontaneously tumored donors ( $(\mathbf{S}$ : Group 0) based on the log-rank statistic indicated that while Group 1 was not significantly different from Group 0 $(p>0.05 ; B)$, Groups 2 and 3 exhibited significantly more rapid tumor development than Groups 0 and 1, with Group 3 also being significantly faster than Group $2(p<0.001)$

the percentage of fish that developed tumors within $10 \mathrm{wk}$ of injection. Group 1 included passages which yielded 5 to $20 \%$, Group 2 yielded 21 to $50 \%$ and Group 3 exhibited greater than 50\% tumored fish within 10 wk of injection (Fig. 2B, Table 2). Based on these criteria, passages 1,2 and 18 were included in Group 1, Passages 4, 5, 7, 14, 16 and 19 were placed in Group 2 and passages 3, 6, 8, 9, 10, 11, 12, 13, 15 and 17 composed Group 3. A comparison of the cumulative frequency curves between these groups and the initial tumors derived from spontaneously tumored donors (Group 0) indicated that while Group 1 was not significantly different from Group 0 (log-rank test, $\mathrm{p}>0.05$; Fig. 2B), Groups 2 and 3 exhibited significantly more rapid tumor development than Groups 0 and 1, with Group 3 also being significantly faster than Group 2
( $\log$-rank test, $p<0.001)$. These results show that most serial passages beyond passage 3 yielded significantly faster tumor development than was obtained by passaging from spontaneous tumors.

Tumors appeared significantly earlier at the posterior left (PL) injection site than at the anterior right (AR) site in all of the serial passage groups as well as the original passages (Fig. 3; log-rank test, $p<0.001$ ). In the few experiments involving intraperitoneal injections, tumor development in this area was significantly slower than at either of the intramuscular sites (logrank test, $\mathrm{p}<0.001$; data not shown). Tumor development at sites remote from the injection sites was observed in a small percentage of the fish in all experiments. However, the incidence of tumors at these other sites never exceeded $10 \%$ at $1 \mathrm{yr}$ in any of the

Table 2. Pomacentrus partitus. Groups of serial passage experiments. Group assignment was made on the basis of proportion of fish with visible tumors at 10 wk post-injection. Group 3 was significantly more rapid than all other groups, Group 2 was significantly faster than Groups 1 and 0 (log-rank statistic, $p<0.001$ ). All statistics based on cumulative proportion of fish developing tumors in each group

\begin{tabular}{|c|c|c|c|c|c|c|}
\hline \multirow{3}{*}{$\begin{array}{l}\text { Experimental group } \\
\text { (passage numbers) }\end{array}$} & \multirow{3}{*}{$\begin{array}{l}\text { Sample size, } \\
\text { fish }\end{array}$} & \multicolumn{2}{|c|}{ Time to tumor (wk) } & \multicolumn{3}{|c|}{ Yield of tumored fish } \\
\hline & & First & $50 \%$ & $\%$ tum & red at & Yield at $52 \mathrm{wk}$ \\
\hline & & tumor & tumored & $10 \mathrm{wk}$ & $52 \mathrm{wk}$ & $N\left(\right.$ censored ${ }^{\mathrm{a}}$ ) \\
\hline Original passages $=$ Group 0 & 43 & 5.1 & 32.7 & 16.3 & 76.1 & $32(1)$ \\
\hline Group $1(1,2,18)$ & 92 & 1.3 & 34.6 & 12.1 & 72.7 & $52(26)$ \\
\hline Group $2(4,5,7,14,16,19)$ & 74 & 1.0 & 16.2 & 29.0 & 93.1 & $59(11)$ \\
\hline Group $3(3,6,8,9,10,11,12,13,15,17)$ & 127 & 1.9 & 7.0 & 61.7 & 95.7 & $118(4)$ \\
\hline
\end{tabular}



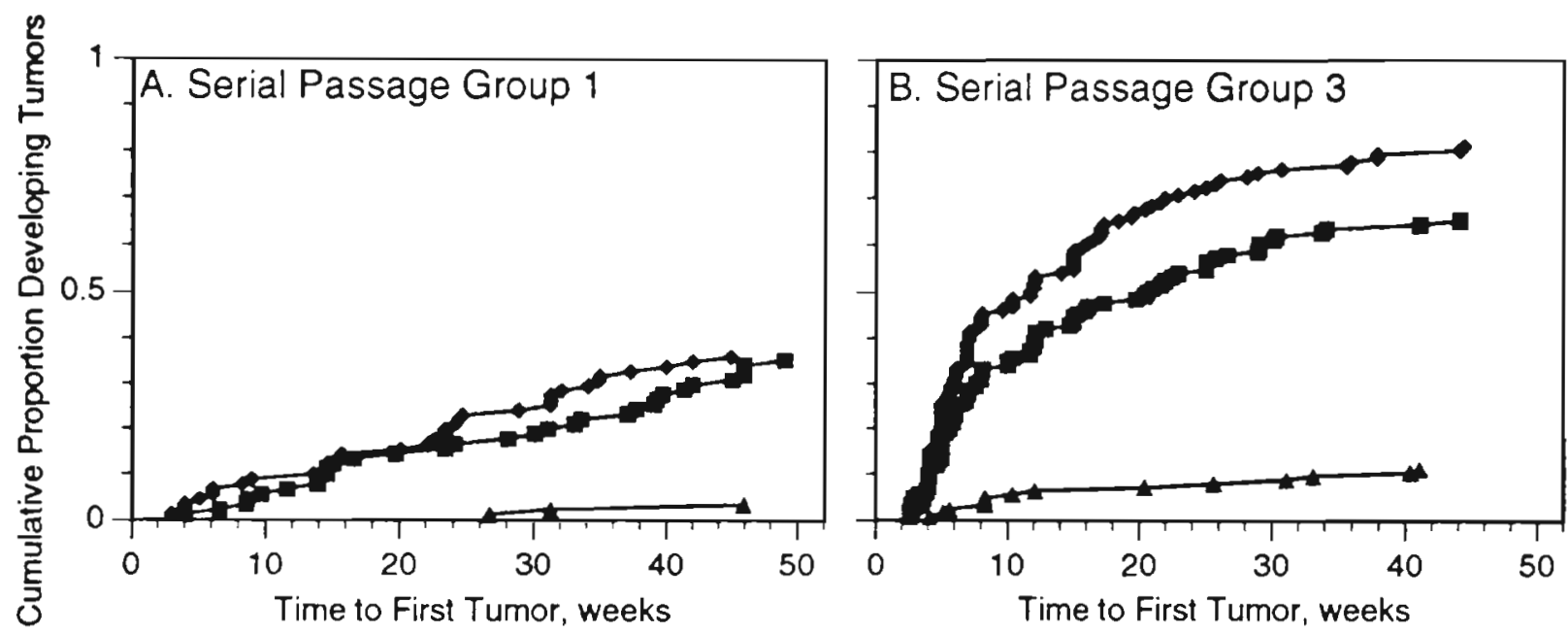

Fig. 3. Pomacentrus partitus. Rates of tumor development by site in serial passage groups. Data from (A) Group 1 and (B) Group 3 are shown as representatives of populations with slowly and rapidly developing tumors, respectively. Tumors appeared significantly faster at the posterior left ( $\bullet$ ) injection site than at the anterior right site ( $)$ in all passage groups ( 0 to 3 ; log-rank statistic, $p<0.001$ ). Tumors at remote sites $(\mathbf{\Lambda})$ only appeared after tumor development at 1 or more injection sites. Tumors at remote sites were most common in Group 3 and least common in Group 1

experimental groups (Fig. 3). In all cases, tumor appearance at AR or PL sites preceded development at remote sites. Tumors at remote sites generally appeared more rapidly after injection in serial passage Groups 2 and 3 (Fig. 3).

The effects of prior exposure or predisposition of different populations to DNF were analyzed by comparing fish collected from areas with high versus low tumor prevalence. Fish were subdivided based on reef of origin into fish collected from a low disease prevalence reef (tumor prevalence $<1 \%$ of adult fish; see Schmale 1991) or one of several high disease prevalence reefs (prevalence $>1 \%$ ). Fish collected from the low disease reef showed a somewhat slower rate of tumor development in serial passage Groups 2 and 3; however, these differences were not significant within any of these groups (log-rank test, $p>0.05$; data not shown).

Growth patterns of induced tumors differed from those of spontaneous tumors in the degree of dissemination, rate of growth, eventual mass of the tumors and mortality rates. In spontaneous cases of DNF, tumors were always widely disseminated through the peripheral nervous system of the fish and were visible externally as hyperpigmented spots or larger, nonpigmented nodules (Fig. 4A; see also Schmale et al. 1983, 1986). In contrast, experimentally induced tumors were generally localized in the region near the injection sites with very little discontinuous spread (Fig. 4A, B, C). No differences were observed in the growth patterns of tumors induced by injection of homogenates of spontaneous tumors (passage Group 0), serially passaged tumors (Groups 1,2 and 3), cell-free filtrates or cultured tumor cells. Induced tumors typically were observed to grow rapidly and extend outward from the injection sites in all directions (Fig. 4). Tumor growth was visible externally as a progressive increase in the surface area and relief of the tumors. Most extemally visible tumors exhibited some hyperpigmentation in the dermal layers due to the presence of increased numbers of melanophores, xanthophores and iridophores (data not shown). This hyperpigmentation occasionally extended deeper into the tumors. Larger tumors often exhibited areas of necrosis in the center of the tumor, likely due to the collapse of the local blood supply due to damage to blood vessels at the outer margins of the tumor (Fig. 4B).

The disseminated distribution of tumors in spontaneous DNF prevented accurate estimation of the total mass of tumors on affected fish, however, we believe that spontaneous tumors rarely exceeded $10 \%$ of body weight (a large adult fish typically weighed about $8 \mathrm{~g}$ ). In contrast, dissection of lesions from juvenile fish with induced tumors revealed that tumors often exceeded $10 \%$ of body mass and occasionally reached $20 \%$ or more within 12 mo of injection (typical juvenile body weights ranged from 2.5 to $6 \mathrm{~g}$ ). Thus, although accurate assessment of growth rates of experimentally induced or spontaneous tumors was not possible, we believe that experimentally induced tumors grew more rapidly and typically attained a larger total mass than spontaneously induced tumors. In addition, experimental fish generally died or required euthanasia (due to tumor development impairing normal behavior) at a greater rate than spontaneously tumored fish in the laboratory (data not shown). 

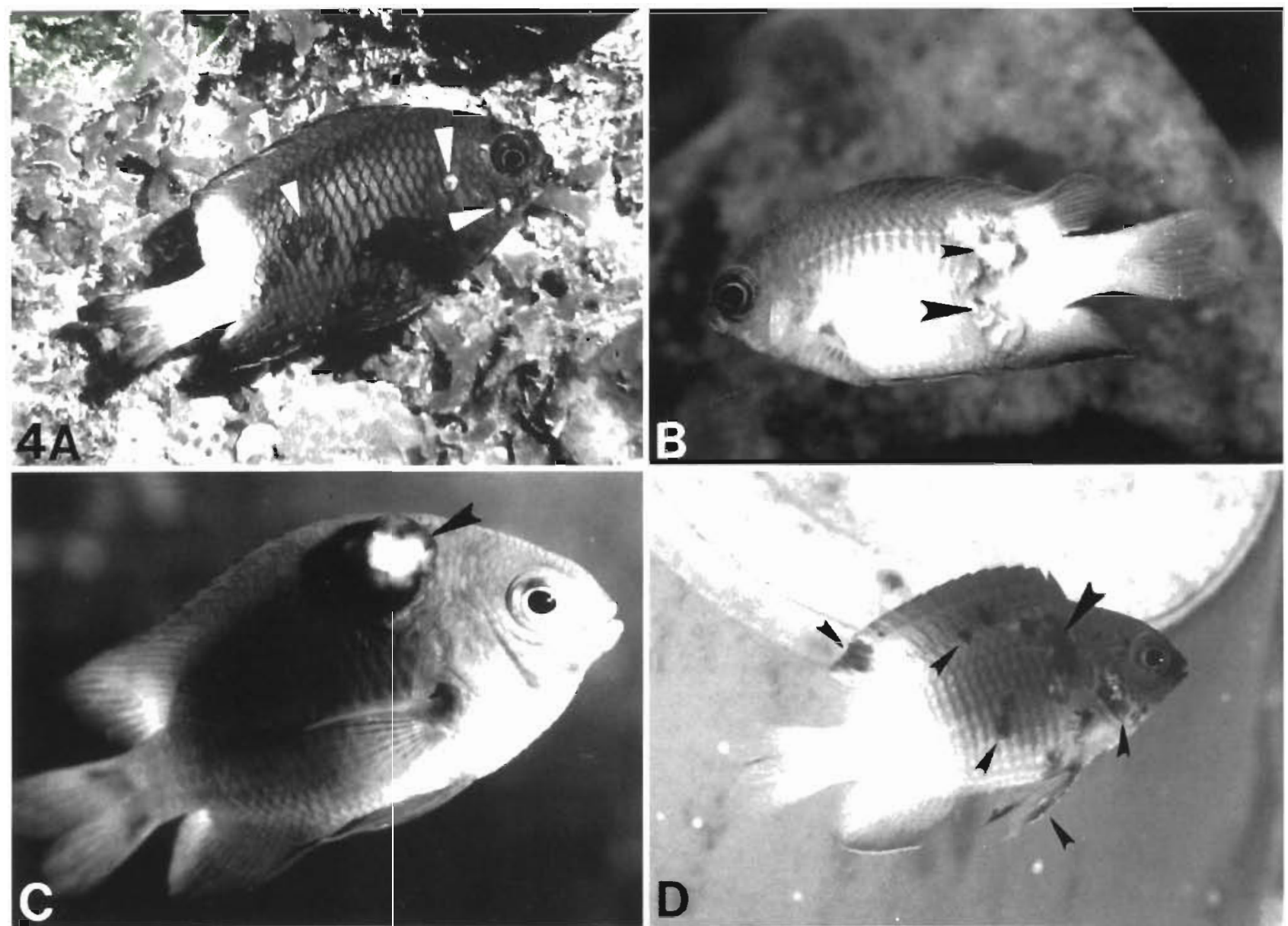

Fig. 4. Pomacentrus partitus. External appearance of spontaneous and experimentally induced tumors. (A) Fish with DNF in the wild (photographed on Molasses Reef, Key Largo, Florida) exhibits widely disseminated pigmented tumors on edges of fins, head region and sides of body (small arrows) as well as non-pigmented, nodular tumors (large arrows) in a pattern typical of spontaneous tumors. (B) Experimentally induced tumors spreading from injection site (located near the dorsal, hyperpigmented region of the tumor; small arrow). An area of necrosis is visible as a small ulceration ventral to this hyperpigmented area (large arrow). (C) Large experimentally induced tumor (arrow) near injection site. (D) Disseminated tumors (small arrows) that have spread discontinuously from the original tumor near the injection site (large arrow)

The histology of all types of induced DNF tumors was indistinguishable from that of spontaneous tumors. Tumors of all types were highly invasive of skin, scales, skeletal muscle, pronephros and bone (Figs. 5 to 7). Less common areas of invasion included the gut, gonads and spleen. Occasionally, induced tumors were observed to invade the spinal canal, a pattern not exhibited by spontaneous tumors. A consistent feature of all lesions was the presence of greatly enlarged nerve fascicles within the tumors, a diagnostic characteristic of plexiform neurofibromas (Figs. 5 to 7). Cytology of the tumors was variable; smaller cells generally had hyperchromatic nuclei while nuclei of larger cells typically exhibited marginated chromatin. The degree of extracellular space within tumors was also variable. The dominant pattern of cell growth was elongate cells arranged in whorling fascicles. These assemblages are typical of those observed in neurofibromas and neurofibrosarcomas in mammals (Harkin \& Reed 1969). Celis containing eosinophilic granules were also a component of all tumors (Vicha \& Schmale 1994).

\section{DISCUSSION}

Viral carcinogenesis has been documented in fishes. Dermal sarcoma in walleye, plasmacytoid leukemia in chinook salmon and lymphosarcoma in pike and muskellunge have been shown to be caused by retroviruses (Papas et al. 1976, Martineau et al. 1991, Eaton \& Kent 1992). Epidermal papillomas in masu salmon and in carp have been shown to be caused by herpesviruses (Anders \& Yoshimizu 1994). In addition, a 
Figs. 5 to 7. Pomacentrus partitus. Comparison of histology of spontaneous and experimentally induced neurofibromas

T: tumor; b: bone; m: muscle; n: nerve; s: scale (all H\&E)

Fig. 5. Spontaneous neurofibroma in isthmus between mandibles. (A) Tumor has replaced most of muscle and some bone and protrudes into the buccal cavity (c) and outward below the jaw of the fish. Enlarged, abnormal nerve is present in center of tumor (arrow). $(\times 20)$. (B) Higher magnification of lesion in the region of the nerve shown in (A) showing several enlarged nerves, pleomorphic tumor cells and bone. $\{\times 200\}$. (C) Higher magnification of adjacent area illustrating the wide variety of cell sizes and morphologies, including highly elongated cells, observed in these tumors. $(\times 400)$

Fig. 6. Tumor induced experimentally by injection of cell-free filtrate of tumor homogenate from the second serial passage. (A) Tumor mass protruding through the dermis and pushing scales outward while also invading the muscle. Enlarged, abnormal nerve is present within tumor (arrow). ( $\times 20)$. (B) Higher magnification of enlarged nerve and the surrounding tumor cells, many of which are elongated and spindle shaped. ( $\times 200)$. (C) Higher magnification of adjacent area illustrating a wide variety of cell sizes and morphologies, including highly elongated cells, and invasion of adjacent myomeres by tumor. $(\times 400)$

Fig. 7. Neurofibroma induced experimentally as a sixteenth serial passage in vivo using unfiltered tumor homogenate. (A) Tumor located in dorsal body musculature. This neurofibroma has not yet penetrated the dermis and the scales on the surface of the body are undisturbed. $(\times 40)$. (B) Higher magnification of enlarged nerve and the surrounding cells, many of which are elongated and spindle shaped. $(\times 200)$. (C) Higher magnification of adjacent area illustrating the wide variety of cell sizes and morphologies, including highly elongated cells. $(\times 400)$

number of other tumor types in a wide variety of fishes are suspected of being caused by viruses (Bowser \& Casey 1993, Anders \& Yoshimizu 1994). However, although peripheral nerve sheath tumors such as neurofibromas, schwannomas and neurofibrosarcomas are relatively common in fishes (Harshbarger et al. 1981), none have been shown to be transmissible or to have a viral etiology

Transmission experiments using cell-free filtrates of tumor homogenates clearly demonstrated that DNF is caused by a subcellular agent. This result rules out the possibility that transmission could be due to transplantation of tumor cells as occurs in the infectious veneral sarcoma of dogs (Moulton 1978) and the reticulum cell sarcoma of Syrian hamsters (Brindley \& Banfield 1961). The continued effectiveness of the serial transmission experiments over many passages also indicates that these tumors are not caused by chemical or physical carcinogens in the environment. Although numerous examples of chemically induced cancers have been reported in fishes, none have been shown to be transplantable (Takayama et al. 1981, Mix 1986). Thus, it is
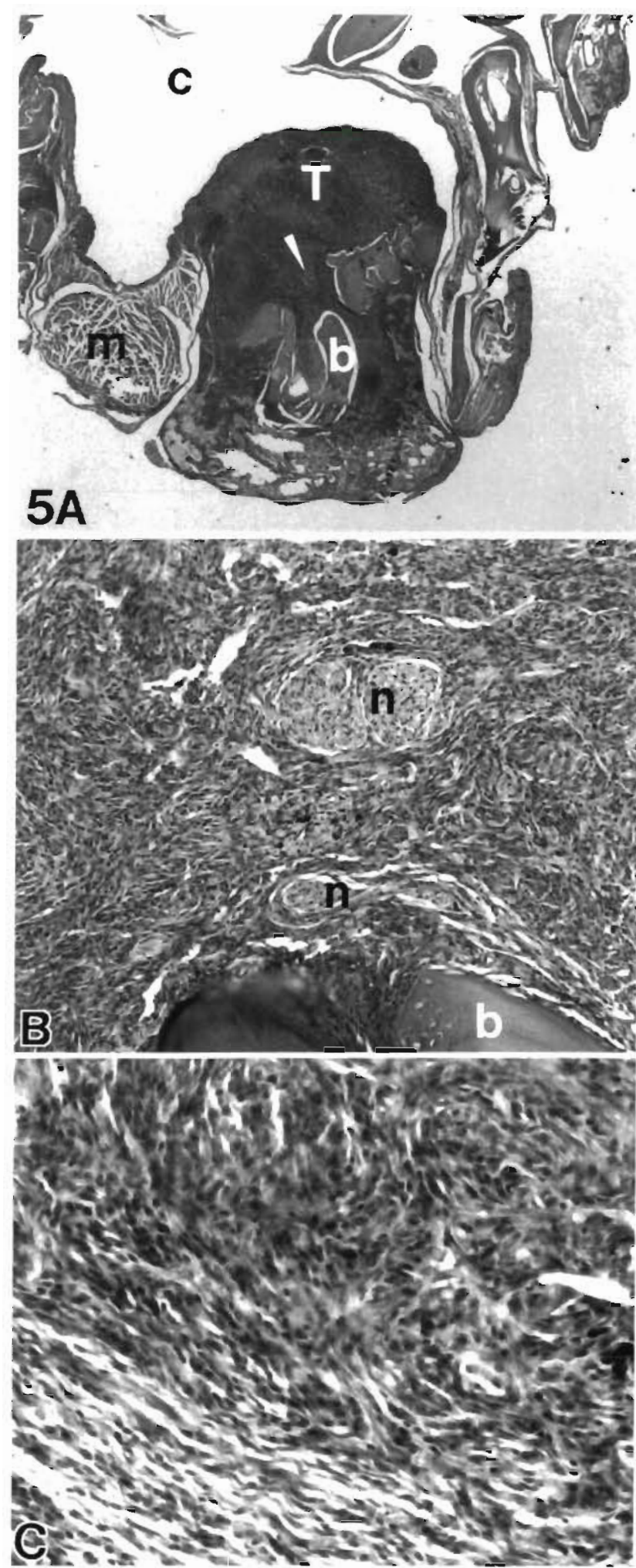

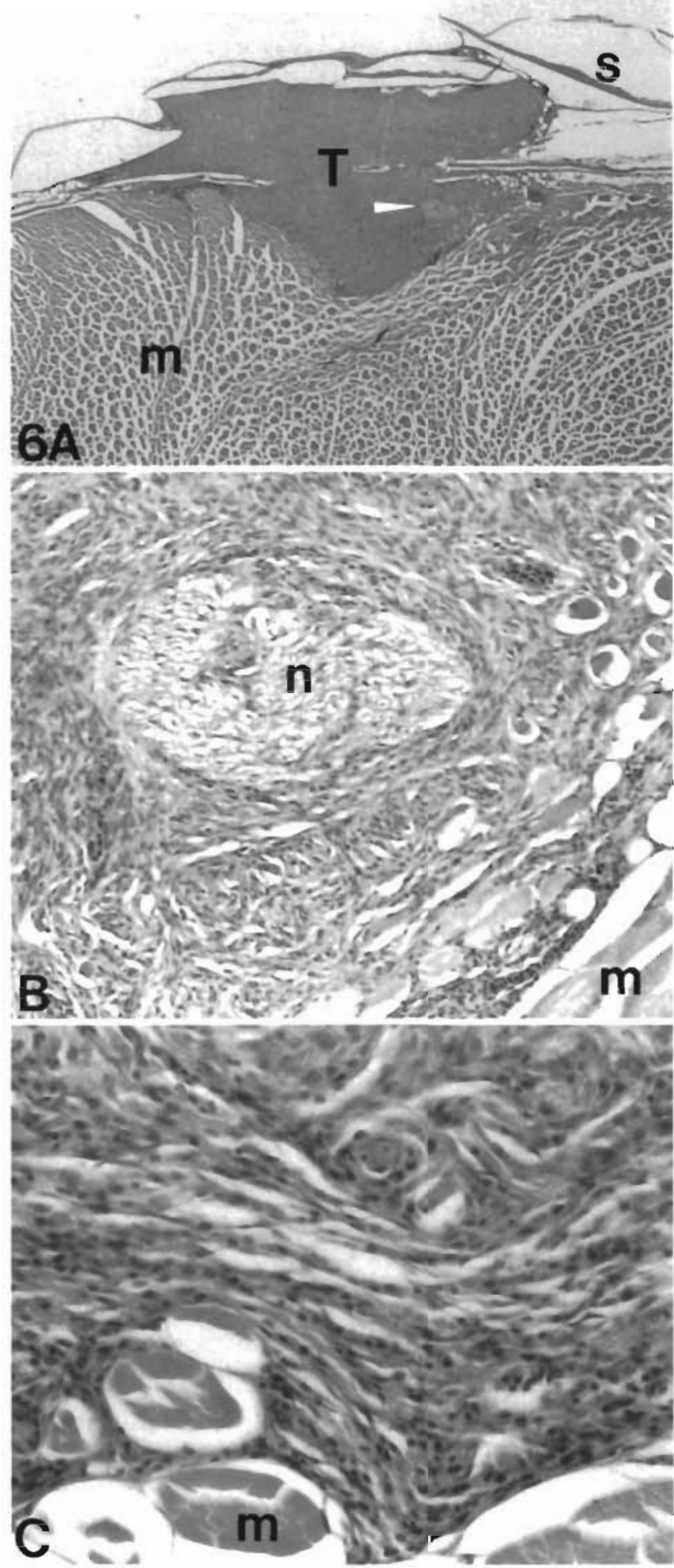
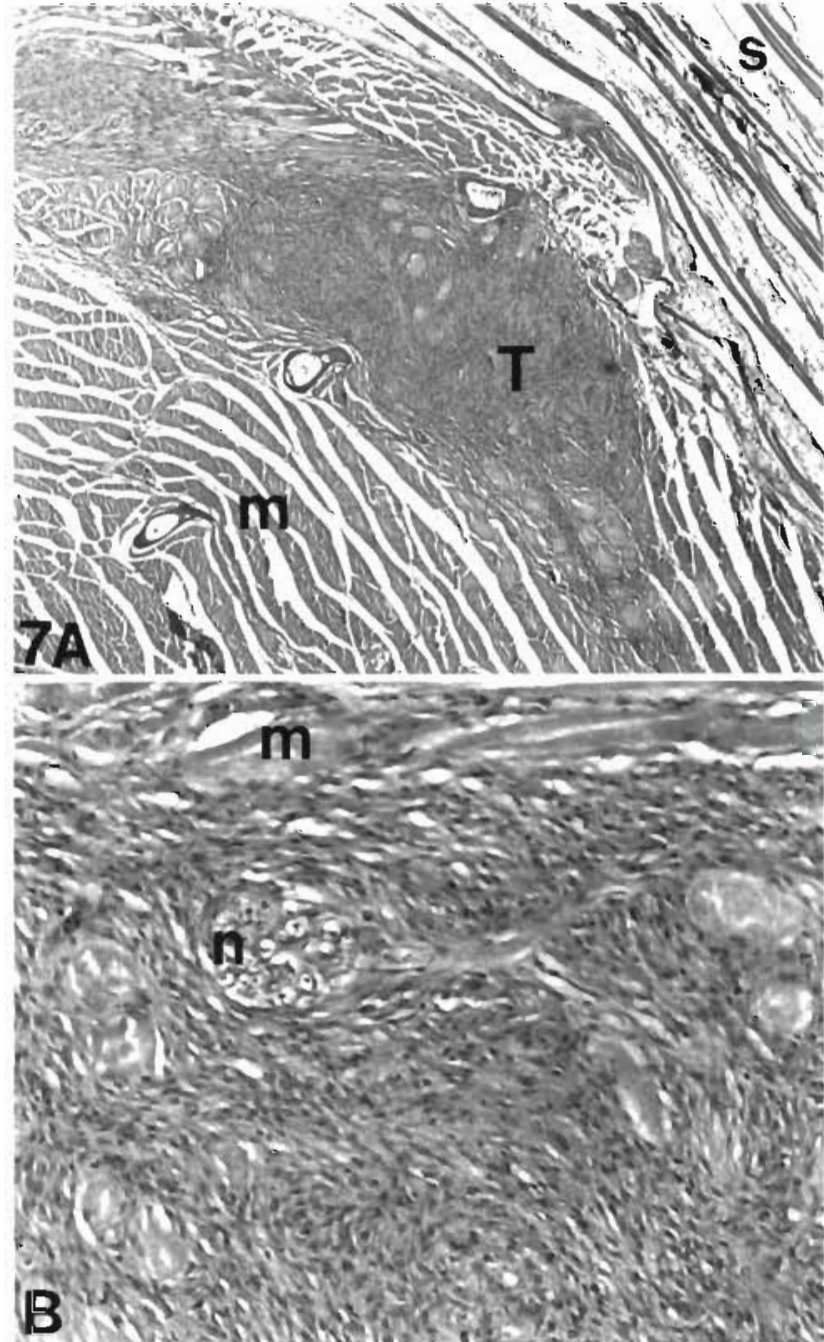
Ays it

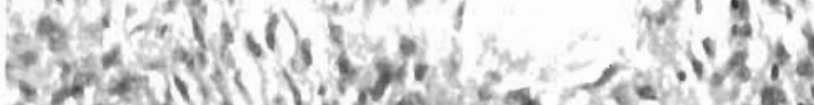

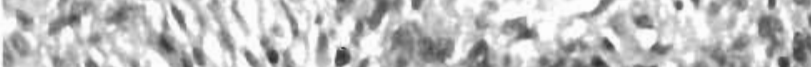

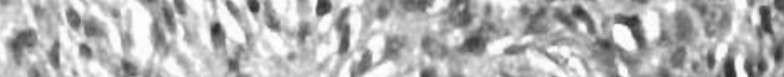

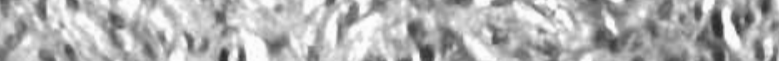

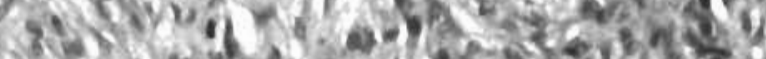

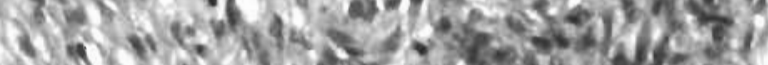

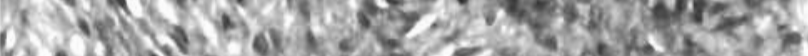

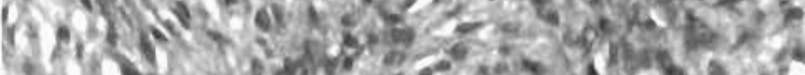

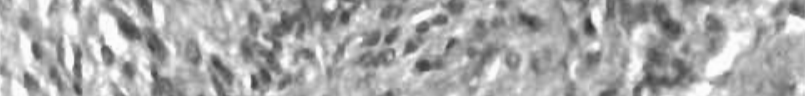

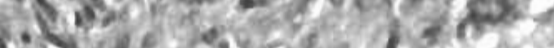

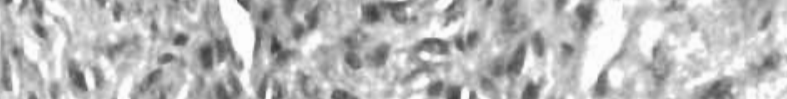

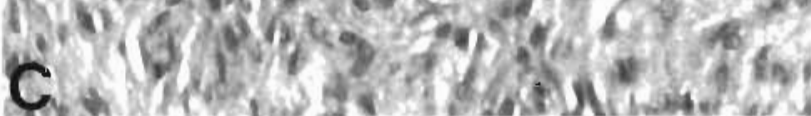


most likely that DNF is caused by a viral agent. Although other agents such as mycoplasmas and prions could have passed through the $0.22 \mu \mathrm{m}$ filters used in these studies. such agents have not been associated with neoplastic diseases. In addition, the tumorigenicity of the DNF tumor cell lines, but not the normal cell cultures or cell line from this species, indicates that the agent responsible for DNF is present in the tumor cell lines.

Differences in genetic predisposition or susceptibility to DNF probably do not affect tumor development rates in the field or in the laboratory. This is suggested by the lack of significant differences observed in development rates of induced tumors in fish from low compared to high tumor prevalence reefs. Furthermore, studies of population genetics of bicolor damselfish in Florida as well as this and other marine fish species in the Southeast Atlantic region have shown a complete lack of any detectable population substructure throughout the entire Caribbean basin, Florida and the Bahamas (Lacson \& Morizot 1991). Thus, there is no evidence to support the involvement of any genetic factors (heritable mechanisms) in DNF. These data also indicate that any increased probability of prior exposure to spontaneous cases of DNF that might have been anticipated for fish from high prevalence reefs does not appear to affect susceptibility of these fish to tumor induction in the laboratory. Such prior exposure could have resulted in the development of spontaneous tumors in these fish, an outcome not observed in this study. In addition, such prior exposure could have increased or decreased the likelihood of tumor development, depending on the relative immunostimulatory and immunosuppressive effects of the etiologic agent.

Several lines of evidence in addition to the cell-free transmission experiments indicate that these tumors are induced by a transmissible agent and are not the result of transplantation of donor cells. Previous studies have indicated that bicolor damselfish are capable of accelerated second-set graft rejection responses (as are other teleost fishes) and that strong allogeneic reactions are exhibited in mixed leukocyte assays between spleen and pronephros cells of individuals from the same reef (McKinney \& Schmale 1992). These findings suggest that these fish are outbred and that allografts would be unlikely to survive. The presence of giant nerves in the plexiform regions of induced neurofibromas is further indication that these tumors arose from transformation of host tissues rather than transplantation of donor cells.

The serial passage experiments demonstrate that repeated in vivo passage of tumors leads to a significant acceleration of tumorigenesis. This result is similar to that observed with a number of retroviruses such as murine leukemia virus (Gross et al. 1963) and
Rous sarcoma virus (Weiss et al. 1982) which exhibit a decrease in latency and a broadening of the host range following serial passage. We have not yet attempted to determine the host range of the spontaneous tumors or whether serial passage increases this range.

The observation that tumors appeared more rapidly at the PL injection site than at the AR site in all of the serial passage groups as well as the original passages was unexpected. This difference may have been due to 2 factors that resulted in easier visualization of tumors at the PL site at an earlier stage of development: tissue thickness and skin color. The thinner layer of musculature present at the more posterior site (as the body tapered toward the caudal peduncle) might have facilitated detection of tumors at a smaller size there than at the more anterior site. Similarly, the skin pigmentation is much lighter in the posterior half of the body in the bicolor damselfish, probably aiding detection of smaller tumors in this region. Studies of intradermal inoculations of mammary adenocarcinomas indicated that more cranial injection locations on the flanks of mice exhibited significantly more rapid rates of tumor development than more caudal sites (Auerbach et al. 1978). The authors suggested that the critical factors determining this difference might be variations in degrees of vascularization and/or innervation of these regions. There is no reason to suspect that tumor development would be influenced by the left or right side location difference in the AR and PL sites, however, we did not conduct experiments to evaluate this factor. Regardless of the reasons for these differences in fish or mice, these observations suggest the importance of carefully standardizing the location of injection, rather than considering large body areas as equivalent, when evaluating tumorigenic potential of any material.

The major phenotypic differences observed between experimentally induced tumors (in all protocols) and spontaneous cases of DNF were in the degree of dissemination of the tumors and their typical rate of growth. In models of transmissible tumors, a variety of features of the inoculum such as source of the tumor extract, route of exposure and dosage as well as host characteristics such as strain and age have been shown to affect growth rates and patterns of virally caused tumors (Harvey \& East 1971, Weiss et al. 1982, Dolberg et al. 1985). In addition, studies of chickens injected with Rous sarcoma virus have shown that tumor development is restricted to the injection site unless wounds are induced at other sites, whereupon tumor formation also occurs at the wound sites (Dolberg et al. 1985, Martins-Green et al. 1994). In the DNF tumor model, induced tumors are always localized, with subsequent discontinuous spread a rare event, and typically are more rapidly growing and more rapidly lethal than naturally occurring tumors. This pattern is indepen- 
dent of the age of the host, although juvenile fish develop tumors in a shorter time after injection than do adults (Schmale \& Hensley 1988). No differences in patterns were observed between fishes receiving intraperitoneal as well as intramuscular injections of tumor homogenates. In addition, type of tumor extract, whether early or late passage tumor homogenate, cellfree filtrate or cultured tumor cells, did not affect growth patterns. Finally, a wide range in concentrations of cultured tumor cells injected ( 1 to $10 \times 10^{6}$ cells $\mathrm{ml}^{-1}$ ) did not produce disseminated tumors. Other injection routes (i.e. intravenous) or higher dosages might have produced more widely disseminated tumors. However, the most likely explanation may be in the nature of the exposure and the response of the host.

A previously proposed mechanism for the spread of DNF in the wild is transmission by direct contact during aggressive interactions that are common in this species (Schmale 1991). These encounters are often associated with minor injuries such as torn fins. When healthy juvenile fish are placed in an aquarium with spontaneously tumored adults, some develop tumors after 6 to 18 mo (Schmale unpubl. data). These tumors appear in a disseminated, relatively slowly progressing pattern that is typical of spontaneous cases of DNF. Thus, the nature of the injection event itself, a delivery of a single concentrated mass of the agent to a single area, may act in several ways to determine the subsequent tumor pattern. The injection may produce sufficient trauma accompanied by inflammation and subsequent cell proliferation to promote tumorigenesis as in the Rous sarcoma-wounding model. An additional possibility is that circumscribed delivery and an initial localization of tumor development provide the maximal opportunity for the host to mount an immune response to the tumor agent. Such a response might be effective in preventing appearance of new tumors, even if it was insufficient or too delayed to prevent or contain the initial tumor. Recent studies of host responses in the DNF model have shown that fish injected with tumor homogenates or cultured cells exhibit a significantly elevated cytotoxicity toward DNF tumor targets (including the same cell lines that induced the tumors; McKinney \& Schmale 1994). In addition, the observation that a few fish in most of the experiments exhibit no tumor development suggests that these individuals may have an immune response of sufficient magnitude to be protective. Preliminary studies of such fish have indicated that they have among the strongest cytotoxic responses of all the injected fish (McKinney \& Schmale unpubl. data). These observations suggest the possibility that immunosuppression of injected fish might result in development of disseminated tumors.

In contrast to the differences in growth patterns observed, the histological phenotype of the spontaneous, wild-type DNF lesions was indistinguishable from that of the induced tumors, regardless of the protocol used for tumor induction (filtered or unfiltered tumor homogenates, serial passages of homogenates or tumor cell lines). This is particularly significant as the later serial passages also represent propagation in vivo for periods as long as $7 \mathrm{yr}$ (to reach the high passage numbers) while the tumor cell lines had been maintained in vitro for up to 70 mo and subcultured up to 45 times before being used to induce tumors in these experiments. Studies in many mammalian systems have revealed significant phenotypic drift of some tumor types after repeated passage in vivo that compromises their usefulness as experimental models for a given tumor type (Kamel et al. 1989). In contrast, the observations reported here indicate that the process of neoplastic transformation is relatively stable in the DNF system with no apparent phenotypic drift caused by these manipulations. This similarity in histology is consistent with the similarities observed in both the antigenic properties of the cell lines derived from spontaneous versus induced tumors (Schmale et al. 1994) and the characteristics of the ion channels in the membranes of cells cultured from these tumor types (Fieber \& Schmale 1994)

Oncogenic DNA and RNA viruses have been associated with a wide variety of tumor types in mammals, birds, reptiles and fishes. However, none has been associated with naturally occurring glial, neural or chromatophore tumors (Tooze 1980. Weiss et al. 1982). Gliomas have been produced experimentally by protocols such as intracerebral inoculation of Rous sarcoma virus (an avian retrovirus) into mice (Kumanishi et al. 1973) while neurofibroma-like tumors have been produced in transgenic mice by insertion of a portion of the tax and LTR regions of HTLV-1 (a human retrovirus) into the genome (Hinricks et al. 1987). However, these tumors are not representative of normal virushost interactions. Given the scarcity of peripheral nerve sheath and chromatophore tumors known to be caused by viruses and the relative abundance in fish and humans of these tumor types, DNF should be an important model for investigating this unique form of viral oncogenesis.

Acknowledgements. The author is grateful to Dr Judy Bean for statistical advice, Dr G. T Hensley for assistance with photomicrography, to Drs Hensley and L. A. Fieber for comments on a draft of the manuscript and to S. M. Cacal, K. A. Gill, S. D. Baribeau and M. W. Greene for assistance in data collection. In addition, thanks are extended to the personnel of the Biscayne National Park. (National Park Service) and the Key Largo National Marine Sanctuary, National Oceanic and Atmospheric Administration (research conducted under permits KLNMS \& LKNMS- 2-86, 3-88, 5-89 and 1-91). This research supported by PHS grants NS21997 and P30 ES05705. 


\section{LITERATURE CITED}

Anders K, Yoshimizu M (1994) Role of viruses in the induction of skin tumours and tumour-like proliferations of fish. Dis aquat Org 19:215-232

Auerbach R, Morrissey LW, Sidky YA (1978) Regional differences in the incidence and growth of mouse tumors following intradermal or subcutaneous inoculation. Cancer Res 38:1739-1744

Bowser PW. Casey JW (1993) Retroviruses of fish. A Rev Fish Dis 209:209-224

Brindley DC, Banfield WG (1961) A contagious tumor of the hamster. J natl Cancer Inst 26:949-957

Dolberg DS, Hollingsworth R, Hertle M, Bissell MJ (1985) Wounding and its role in RSV-mediated tumor formation. Science 230:676-678

Eaton WD, Kent ML (1992) A retrovirus in chinook salmon (Oncorhynchus tshawytscha) with plasmacytoid leukemia and evidence for the etiology of the disease. Cancer Res $52: 1-6$

Elandt-Johnson RC, Johnson NL (1980) Survival models and data analysis. Wiley and Sons, New York

Fieber LA, Schmale MC (1994) Differences in a K current in Schwann cells from normal and neurofibromatosisinfected damselfish. Glia 11:64-72

Gross L, Dreyfuss Y, Morre LA (1963) Serial cell-free passage in rats of the mouse leukemia virus: effect of thymectomy. Proc Soc exp Biol Med 112:939-945

Harkin JC, Reed RJ (1969) Tumors of the peripheral nervous system. Armed Forces Institute of Pathology, Washington, DC

Harshbarger JC, Charles AM, Spero PM (1981) Collection and analysis of neoplasms in sub-homeothermic animals from a phyletic point of view. In: Dawe CJ, Harshbarger JC, Kondo S, Sugimura T, Takayama S (eds) Phyletic approaches to cancer Japan Scientific Societies Press, Tokyo, p $357-385$

Harvey JJ, East J (1971) The murine sarcoma (MSV). Int Rev exp Pathol 10:265-360

Hinricks SH, Nerenberg M, Reynolds RK, Khoury G, Jay G (1987) A transgenic mouse model for human neurofibromatosis. Science 237:1340-1344

Kamel HMH, Willmott N, McNicol AM, Toner PG (1989) The use of electron microscopy and immunocytochemistry to characterise spontaneously-arising, transplantable rat tumors. Virchows Arch B Cell Pathol 57:11-18

Kumanishi T, lkuta F. Yamamoto T (1973) Brain tumors induced by Rous sarcoma virus, Schmidt-Ruppin strain. III. Morphology of brain tumors induced in adult mice. $J$ natl Cancer Inst 50:95-109

Lacson JM, Morizot DC (1991) Temporal genetic variation in subpopulations of bicolor damselfish (Stegastes partitus) unhabiting coral reefs in the Florida Keys. Mar Biol 110: $353-357$

Responsible Subject Editor: N. Peters, Hamburg. Germany
Martineau D, Renshaw R, Williams JR, Casey JW, Bowser PR (1991) A large unintegrated retrovirus DNA species present in a dermal tumor of walleye Stizostedion vitreum. Dis aquat Org 10:153-158

Martins-Green M, Boudreau N, Bissell MJ (1994) Inflammation is responsible for the development of wound-induced tumors in chickens infected with Rous Sarcoma Virus. Cancer Res 54:4334-4341

McKinney EC, Schmale MC (1992) Primed allogeneic reactions to leukocytes and epithelial cells in the bicolor damselfish. Transplantation 54:31.3-317

McKinney EC, Schmale MC (1994) Damselfish with neurofibromatosis exhibit cytotoxicity toward tumor targets. Dev comp Immunol 18:305-313

Mix MC (1986) Cancerous diseases in aquatic animals and their association with environmental pollutants: a critical literature review. Mar environ Res 20:1-111

Moulton JE (ed) (1978) Tumors in domestic animals. University of California Press. Berkeley

Papas TS, Dahlberg JE, Sonstegard RA (1976) Type C virus in lymphosarcoma in northern pike (Esox lucius). Nature 261:506-508

Schmale MC (1991) Prevalence and distribution patterns of tumors in bicolor damselfish (Pomacentrus partitus) on South Flonda reefs. Mar Biol 109:203-21.2

Schmale MC, Gill KA, Cacal SM, Baribeau SD (1994) Characterization of Schwann cells from normal nerves and from neurofibromas in the bicolor damselfish. J Neurocytol 23 : 668-681

Schmale MC, Hensley GT (1988) Transmissibility of a neurofibromatosis-like disease in bicolor damselfish. Cancer Res 48:3828-3833

Schmale MC, Hensley GT, Udey LR (1983) Multiple schwannomas in the bicolor damselfish, Pomacentrus partitus; a possible model of von Recklinghausen neurofibromatosis. Am J Pathol 112:238-241

Schmale MC, Hensley GT, Udey LR (1986) Neurofibromatosis in the bicolor damselfish (Pomacentrus partitus) as a model of von Recklinghausen neurofibromatosis. Annls NY Acad Sci 486:386-402

Takayama S, Ishikawa T, Masahito P, Matsumoto J (1981) Overview of biological characterization of tumors in fish. In: Dawe CJ, Harshbarger JC, Kondo S, Sugimura $T$, Takayama S (eds) Phyletic approaches to cancer Japan Scientific Societies Press, Tokyo, p 3-18

Tooze J (ed) (1980) DNA tumor viruses. Cold Spring Harbor Publ., Cold Spring Harbor, NY

Vicha DL, Schmale MC (1994) Morphology and distribution of eosinophilic granulocytes in damselfish neurofibromatosis, a model of mast cell distribution in neurofibromatosis type 1. Anticancer Res 14:947-952

Weiss R, Teich N, Varmus H, Coffin J (1982) RNA tumor viruses. Cold Spring Harbor Publ, Cold Spring Harbor, NY

Manuscript first received: March 22, 1995

Revised version accepted: June 13, 1995 